\title{
A Study of Hexagon Star Network with Vertex-Edge-Based Topological Descriptors
}

\author{
Eshrag A. Refaee (D) and Ali Ahmad (iD \\ College of Computer Science \& Information Technology Jazan University, Jazan, Saudi Arabia \\ Correspondence should be addressed to Eshrag A. Refaee; erefaie@jazanu.edu.sa
}

Received 22 March 2021; Revised 5 April 2021; Accepted 13 April 2021; Published 27 April 2021

Academic Editor: Muhammad Javaid

Copyright (c) 2021 Eshrag A. Refaee and Ali Ahmad. This is an open access article distributed under the Creative Commons Attribution License, which permits unrestricted use, distribution, and reproduction in any medium, provided the original work is properly cited.

\begin{abstract}
There are many network topology designs that have emerged to fulfill the growing need for networks to provide a robust platform for a wide range of applications like running businesses and managing emergencies. Amongst the most famous network topology designs are star network, mesh network, hexagonal network, honeycomb network, etc. In a star network, a central computer is linked with various terminals and other computers over point-to-point lines. The other computers and terminals are directly connected to the central computer but not to one another. As such, any failure in the central computer will result in a failure of the entire network and computers in star network will not be able to communicate. The star topology design can be represented by a graph where vertices represent the computer nodes and edges represent the links between the computer nodes. In this paper, we study the vertex-edge-based topological descriptor for a newly designed hexagon star network.
\end{abstract}

\section{Introduction}

The world of information technology has been witnessing the evolvement of computer networks to serve many different real-life applications. As such, the evolvement of robust networks has become vital to provide a platform on which modern communications occur. Direct interconnection networks can be modeled by graphs, with nodes and edges corresponding to computer nodes and communication links between them, respectively. In [1], the authors present a survey of summarizing the pros and cons of these types of networks. A network topology like star network suffers from its vulnerability of having all computers nodes linked to a central computer node which as a result can cause the entire network to fail to communicate once a failure occurs in the central computer node.

In a fixed interconnection parallel architecture, vertices represent computer nodes while edges represent communication links between nodes. Researchers in parallel processing are motivated to utilize a new improved interconnection network wherein performance is evaluated under different variety of circumstances. Networks like hexagonal, honeycomb, and grid networks have interesting topological properties, such as bearing resemblance to atomic or molecular lattice structures [2]. Such a property allows flexibility in using the aforementioned networks in variety of different architecture designs for assessing performance in different contexts.

Cheminformatics is a recently evolving multidisciplinary subject, representing the intersection point of chemistry, mathematics, and information science. Graph theory has been heavily utilized in chemistry. In particular, chemical graph theory is the topology branch of mathematical chemistry that implements graph theory to mathematical modeling of chemical occurrence [3-5]. This theory contributes a major role in the domain of chemical sciences.

A topological index is a numeric amount related with a chemical constitution asserting a connection between chemical structures with numerous physicosynthetic properties and chemical reactivity. In this unique circumstance, topological indices are planned with the possibility of change of a chemical structure into a number that portrays the geography of that structure. Topological index has been utilized in science by Wiener in the investigation of paraffin 
breaking points [6], which has been followed by several attempts to clarify physicochemical properties, resulting in presenting many different topological descriptors. For detailed study of topological descriptors, see [7-23]. Chellali et al. [24] presented new degree ideas, namely, "ve-degree and ev-degree." The connection between "traditional degreebased" and "ve-degree and ev-degree" can be seen in [25-30].

\section{Preliminaries}

Let $E$ and $V$ be the edge set and vertex set of a simple connected graph $G=(V, E)$. The degree of a vertex $\theta$, denoted by $\Psi(\theta)$, is the number of edges that are incident to $\theta$. The open neighborhood of a vertex $\theta$, denoted by $N(\theta)$, is a number of all vertices adjacent to $\theta$. The closed neighborhood of $\theta$, denoted by $N[\theta]$, is the union of $\theta$ and $N(\theta)$. The ev-degree, denoted by $\Psi_{\mathrm{ev}}(e)$, of any edge $e=\theta \vartheta \in E$ is the total number of vertices of the closed neighborhood union of $\theta$ and $\vartheta$. The ve-degree, denoted by $\Psi_{\mathrm{ve}}(\theta)$, of any vertex $\theta \in V$ is the number of different edges that are incident to any vertex from the closed neighborhood of $\theta$. For details, see $[24,25,27,28]$. The ve-degree- and ev-degreebased topological indices are defined in the following equations.

$$
\begin{aligned}
\mathbb{M}_{\mathrm{ev}}(G) & =\sum_{\vartheta \in E} \Psi_{\mathrm{ev}}(\theta)^{2}, \\
\mathbb{M}_{\alpha \mathrm{ve}}^{1}(G) & =\sum_{\vartheta \in V} \Psi_{\mathrm{ve}}(\vartheta)^{2}, \\
\mathbb{M}_{\beta \mathrm{ve}}^{1}(G) & =\sum_{\theta \vartheta \in E}\left(\Psi_{\mathrm{ve}}(\theta)+\Psi_{\mathrm{ve}}(\vartheta)\right), \\
\mathbb{M}_{\mathrm{ve}}^{2}(G) & =\sum_{\theta \vartheta \in E}\left(\Psi_{\mathrm{ve}}(\theta) \times \Psi_{\mathrm{ve}}(\vartheta)\right), \\
\mathbb{R}_{\mathrm{ve}}(G) & =\sum_{\theta \vartheta \in E}\left(\Psi_{\mathrm{ve}}(\theta) \times \Psi_{\mathrm{ve}}(\vartheta)\right)^{-(1 / 2)}, \\
\mathbb{R}_{\mathrm{ev}}(G) & =\sum_{\vartheta E} \Psi_{\mathrm{ve}}(\vartheta)^{-(1 / 2)}, \\
\mathbb{X}_{\mathrm{ve}}(G) & =\sum_{\theta \vartheta \in E}\left(\Psi_{\mathrm{ve}}(\theta)+\Psi_{\mathrm{ve}}(\vartheta)\right)^{-(1 / 2)}, \\
\mathbb{A} \mathbb{B} \mathbb{C}_{\mathrm{ve}}(G) & =\sum_{\theta \vartheta \in E}\left(\frac{\Psi_{\mathrm{ve}}(\theta)+\Psi_{\mathrm{ve}}(\vartheta)-2}{\Psi_{\mathrm{ve}}\left(\theta \times \Psi_{\mathrm{ve}}(\vartheta)\right.}\right) \\
\mathbb{G A}_{\mathrm{ve}}(G) & =\sum_{\theta \vartheta \in E} \frac{2\left(\Psi_{\mathrm{ve}}(\theta) \times \Psi_{\mathrm{ve}}(\vartheta)\right)^{(1 / 2)}}{\Psi_{\mathrm{ve}}(\theta)+\Psi_{\mathrm{ve}}(\vartheta)},
\end{aligned}
$$

Equations (1)-(10) are known as ev-degree Zagreb $\alpha$ index, first ve-degree Zagreb $\alpha$ index, first ve-degree Zagreb $\beta$ index, second ve-degree Zagreb index, ve-degree Randic index, ev-degree Randic index, ve-degree atom-bond connectivity index, ve-degree geometric-arithmetic index, ve-degree harmonic index, and ve-degree sum-connectivity index, respectively.

\section{The Hexagon Star Network and Its Applications}

Hexagonal networks belong to the family of networks modeled by planar graphs. There are three regular plane tessellations composed of the same kind of regular (equilateral) polygons: triangular, square, and hexagon. They formed the basis for designing the direct interconnection networks with highly competitive overall performance. Hexagonal networks are based on triangular plane tessellation or the partition of a plane into equilateral triangles provided that each node has up to six neighbors. The closest networks, in terms of design and structure, are those based on regular hexagons, called honeycomb networks. Those based on a regular square partition are called mesh networks $[31,32]$.

A hexagonal network has a shape that is most suitable for cellular communication design of mobile networks for many reasons. In a hexagon grid, the frequencies overlapping from one cell to the other can be clearly seen. In this context, the hexagonal network is proposed as a viable alternative interconnection network to mesh connected computer, with nodes serving as processors. It is also to model cellular networks where nodes represent base stations. In this paper, we define a new interconnection network hexagon star network. The proposed network is a composition of triangles around a hexagon, as shown in Figure 1.

\section{Main Results}

In this section, we determine the ev-degree Zagreb index, first ve-degree Zagreb $\alpha(\beta)$ index, second ve-degree Zagreb $\beta$ index, ve-degree atom-bond connectivity $\left(\mathbb{A} B \mathbb{C}_{\mathrm{ve}}\right)$ index, ve-degree geometric-arithmetic $\left(\mathbb{G A}_{\mathrm{ve}}\right)$ index, ve-degree Randic index, ev-degree Randic index, ve-degree sumconnectivity $\left(\chi_{\mathrm{ve}}\right)$ index, and ve-degree harmonic $\left(\mathbb{H}_{\mathrm{ve}}\right)$ for hexagon star network $G_{l, w}$.

4.1. ev-Degree Zagreb Index. Using Table 1, we compute the ev-degree Zagreb index:

$$
\begin{aligned}
\mathbb{M}_{\mathrm{ev}}\left(G_{l, w}\right) & =\sum_{e_{1} \in E} \Psi_{\mathrm{ev}}\left(e_{1}\right)^{2} \\
\mathbb{M}_{\mathrm{ev}}\left(G_{l, w}\right) & =(8 l+4 w)(6)^{2}+(12 l w-2 l-4 w)(8)^{2} \\
& =768 l w+160 l-112 w .
\end{aligned}
$$

4.2. First ve-Degree Zagreb $\alpha$ Index. Using Table 2, we compute the first ve-degree Zagreb $\alpha$ index: 


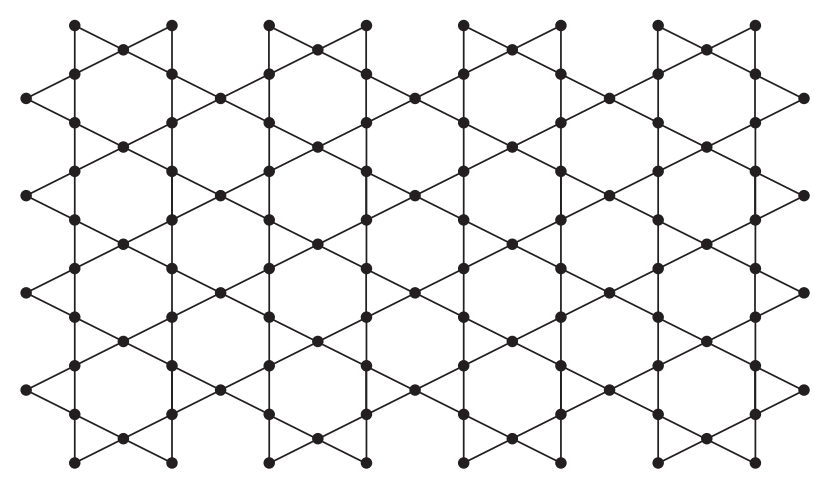

Figure 1: The hexagon star network sheet for $l=4$ and $w=4$.

TABLE 1: ev-Degree of $G_{l, w}$.

\begin{tabular}{lcc}
\hline$(\Psi(\theta), \Psi(\vartheta))$ & ev-Degree & Frequency \\
\hline$(2,4)$ & 6 & $8 l+4 w$ \\
$(4,4)$ & 8 & $12 l w-2 l-4 w$ \\
\hline
\end{tabular}

TABle 2: ve-Degree of $G_{l, w}$.

\begin{tabular}{lcc}
\hline$\Psi(\theta)$ & ve-Degree & Frequency \\
\hline 2 & 8 & $4 l+2 w$ \\
4 & 12 & $2 l+4$ \\
4 & 14 & $4 l+4 w-8$ \\
4 & 16 & $6 l w-5 l-5 w+4$ \\
\hline
\end{tabular}

$$
\begin{aligned}
\mathbb{M}_{\alpha \mathrm{ve}}^{1}\left(G_{l, w}\right)= & \sum_{\theta \in V} \Psi_{\mathrm{ve}}(\theta)^{2} \\
\mathbb{M}_{\alpha \mathrm{ve}}^{1}\left(G_{l, w}\right)= & (4 l+2 w)(8)^{2}+(2 l+4)(12)^{2} \\
& +(4 l+4 w-8)(14)^{2}+(6 l w-5 l-5 w+4)(16)^{2} \\
= & 1536 l w+48 l-368 w+32 .
\end{aligned}
$$

4.3. First ve-Degree Zagreb $\beta$ Index. Using Table 3, we compute the first ve-degree Zagreb $\beta$ index:

$$
\begin{aligned}
\mathbb{M}_{\beta \mathrm{ve}}^{1}\left(G_{l, w}\right)= & \sum_{\theta \vartheta \in E}\left(\Psi_{\mathrm{ve}}(\theta)+\Psi_{\mathrm{ve}}(\vartheta)\right) \\
M_{\beta \mathrm{ve}}^{1}\left(G_{l, w}\right)= & (4 l+8)(20)+(4 l+4 w-8)(22)+(4)(24) \\
+(4 l)(26)= & +(4 w-6)(28) \\
& +(8 l+4 w-12)(30) \\
& +(12 l w-14 l-12 w+14)(32) \\
= & 384 l w+64 l-64 w .
\end{aligned}
$$

4.4. Second ve-Degree Zagreb Index. Using Table 3, we compute the second ve-degree Zagreb index:
TAвLe 3: End vertices' ve-degrees of each edge of $G_{l, w}$.

\begin{tabular}{lcc}
\hline$(\Psi(\theta), \Psi(\vartheta))$ & ve-Degree & Frequency \\
\hline$(2,4)$ & $(8,12)$ & $4 l+8$ \\
$(2,4)$ & $(8,14)$ & $4 l+4 w-8$ \\
$(4,4)$ & $(12,12)$ & 4 \\
$(4,4)$ & $(12,14)$ & $4 l$ \\
$(4,4)$ & $(14,14)$ & $4 w-6$ \\
$(4,4)$ & $(14,16)$ & $8 l+4 w-12$ \\
$(4,4)$ & $(16,16)$ & $12 l w-14 l-12 w+14$ \\
\hline
\end{tabular}

$$
\begin{aligned}
\mathbb{M}_{\mathrm{ve}}^{2}\left(G_{l, w}\right)= & \sum_{\theta \vartheta \in E}\left(\Psi_{\mathrm{ve}}(\theta) \times \Psi_{\mathrm{ve}}(\vartheta)\right), \mathbb{M}_{\mathrm{ve}}^{2}\left(G_{l, w}\right) \\
= & (4 l+8)(96)+(4 l+4 w-8)(112)+(4)(144) \\
& +(4 l)(168)+(4 w-6)(196) \\
& +(8 l+4 w-12)(224) \\
& +(12 l w-14 l-12 w+14)(256) \\
= & 3072 l w-288 l-944 w+168 .
\end{aligned}
$$

4.5. ve-Degree Randic Index. Using Table 3, we compute the ve-degree Randic index:

$$
\begin{aligned}
\mathbb{R}_{\mathrm{ve}}\left(G_{l, w}\right)= & \sum_{\theta \vartheta \in E}\left(\Psi_{\mathrm{ve}}(\theta) \times \Psi_{\mathrm{ve}}(\vartheta)\right)^{-(1 / 2)}, \\
\mathbb{R}_{\mathrm{ve}}\left(G_{l, w}\right)= & (4 l+8)(96)^{-(1 / 2)}+(4 l+4 w-8)(112)^{-(1 / 2)} \\
& +(4)(144)^{-(1 / 2)} \\
& +(4 l)(168)^{-(1 / 2)}+(4 w-6)(196)^{-(1 / 2)} \\
& +(8 l+4 w-12)(224)^{-(1 / 2)} \\
& +(12 l w-14 l-12 w+14)(256)^{-(1 / 2)} \\
= & \frac{3}{4} l w+\left(\frac{1}{6} \sqrt{6}+\frac{1}{21} \sqrt{42}+\frac{1}{7} \sqrt{7}+\frac{1}{7} \sqrt{14}-\frac{7}{8}\right) l \\
& +\left(\frac{1}{7} \sqrt{7}+\frac{1}{14} \sqrt{14}-\frac{13}{28}\right) w \\
& +\frac{1}{3} \sqrt{6}-\frac{2}{7} \sqrt{7}-\frac{3}{14} \sqrt{14}+\frac{131}{168} .
\end{aligned}
$$


4.6. ev-Degree Randic Index. Using Table 1, we compute the ev-degree Randic index:

$$
\begin{aligned}
\mathbb{R}_{\mathrm{ev}}\left(G_{l, w}\right) & =\sum_{e_{1} E} \Psi_{\mathrm{ve}}\left(e_{1}\right)^{-(1 / 2)} \\
R_{\mathrm{ev}}\left(G_{l, w}\right) & =(8 l+4 w)(6)^{-(1 / 2)}+(12 l w-2 l-4 w)(8)^{-(1 / 2)} \\
& =3 \sqrt{2} l w+\left(\frac{4}{3} \sqrt{6}-\frac{1}{2} \sqrt{2}\right) l+\left(\frac{2}{3} \sqrt{6}-\sqrt{2}\right) w
\end{aligned}
$$

4.7. ve-Degree Atom-Bond Connectivity Index. Using Table 3, we compute the ve-degree atom-bond connectivity index:

$$
\begin{aligned}
A \mathbb{B C}_{\mathrm{ve}}\left(G_{l, w}\right)= & \sum_{\theta \vartheta \in E}\left(\frac{\Psi_{\mathrm{ve}}(\theta)+\Psi_{\mathrm{ve}}(9)-2}{\Psi_{\mathrm{ve}}(\theta) \times \Psi_{\mathrm{ve}}(9)}\right)^{(1 / 2)}, \\
\mathbb{A B C} \mathbb{v}_{\mathrm{ve}}\left(G_{l, w}\right)= & (4 l+8) \sqrt{\frac{18}{96}}+(4 l+4 w-8) \sqrt{\frac{20}{112}}+(4) \sqrt{\frac{22}{144}} \\
& +(4 l) \sqrt{\frac{24}{168}}+(4 w-6) \sqrt{\frac{26}{196}} \\
& +(8 l+4 w-12) \sqrt{\frac{28}{224}}+(12 l w-14 l-12 w+14) \sqrt{\frac{30}{256}} \\
= & \frac{3}{4} \sqrt{30} l w+\left(\sqrt{3}+\frac{2}{7} \sqrt{35}+2 \sqrt{2}+\frac{4}{7} \sqrt{7}-\frac{7}{8} \sqrt{30}\right) l \\
& +\left(-\frac{3}{4} \sqrt{30}+\sqrt{2}+\frac{2}{7} \sqrt{35}+\frac{2}{7} \sqrt{26}\right) w \\
& +2 \sqrt{3}+\frac{7}{8} \sqrt{30}-\frac{4}{7} \sqrt{35}-\frac{3}{7} \sqrt{26}-3 \sqrt{2}+\frac{1}{3} \sqrt{22} .
\end{aligned}
$$

4.8. ve-Degree Geometric-Arithmetic Index. Using Table 3, we compute the ve-degree geometric-arithmetic index:

$$
\begin{aligned}
\mathbb{G A}_{\mathrm{ve}}\left(G_{l, w}\right)= & \sum_{\theta \vartheta \in E} \frac{2\left(\Psi_{\mathrm{ve}}(\theta) \times \Psi_{\mathrm{ve}}(\vartheta)\right)^{(1 / 2)}}{\Psi_{\mathrm{ve}}(\theta)+\Psi_{\mathrm{ve}}(\vartheta)} \\
\mathbb{G A}_{\mathrm{ve}}\left(G_{l, w}\right)= & (4 l+8) \frac{(2) \sqrt{96}}{20}+(4 l+4 w-8) \frac{(2) \sqrt{112}}{22} \\
& +(4) \frac{(2) \sqrt{144}}{24}+(4 l) \frac{(2) \sqrt{168}}{26} \\
& +(4 w-6) \frac{(2) \sqrt{196}}{28}+(8 l+4 w-12) \frac{(2) \sqrt{224}}{30} \\
& +(12 l w-14 l-12 w+14) \frac{(2) \sqrt{256}}{32} \\
= & 12 l w+\left(\frac{8}{5} \sqrt{6}+\frac{8}{13} \sqrt{42}+\frac{16}{11} \sqrt{7}+\frac{32}{15} \sqrt{14}-14\right) l \\
& \cdot\left(\frac{16}{11} \sqrt{7}+\frac{16}{15} \sqrt{14}-8\right) w+\frac{16}{5} \sqrt{6} \\
& -\frac{32}{11} \sqrt{7}-\frac{16}{5} \sqrt{14}+12 .
\end{aligned}
$$

4.9. ve-Degree Harmonic Index. Using Table 3, we compute the ve-degree harmonic index:

$$
\begin{aligned}
\mathfrak{H}_{\mathrm{ve}}\left(G_{l, w}\right)= & \sum_{\theta \vartheta \in E} \frac{2}{\Psi_{\mathrm{ve}}(\theta)+\Psi_{\mathrm{ve}}(\vartheta)}, \\
\mathbb{F}_{\mathrm{ve}}\left(G_{l, w}\right)= & (4 l+8) \frac{2}{20}+(4 l+4 w-8) \frac{2}{22}+(4) \frac{2}{24} \\
& +(4 l) \frac{2}{26}+(4 w-6) \frac{2}{28}+(8 l+4 w-12) \frac{2}{30} \\
& +(12 l w-14 l-12 w+14) \frac{2}{32} \\
= & \frac{3}{4} l w+\frac{12521}{17160} l+\frac{767}{4620} w+\frac{97}{1848} .
\end{aligned}
$$

4.10. ve-Degree Sum-Connectivity Index. Using Table 3, we compute the ve-degree sum-connectivity index:

$$
\begin{aligned}
\chi_{\mathrm{ve}}\left(G_{l, w}\right)= & \sum_{\theta \vartheta \in E}\left(\Psi_{\mathrm{ve}}(\theta)+\Psi_{\mathrm{ve}}(\vartheta)\right)^{-(1 / 2)} \\
\chi_{\mathrm{ve}}\left(G_{l, w}\right)= & (4 l+8) \frac{1}{\sqrt{20}}+(4 l+4 w-8) \frac{1}{\sqrt{22}}+(4) \frac{1}{\sqrt{24}} \\
& +(4 l) \frac{1}{\sqrt{26}}+(4 w-6) \frac{1}{\sqrt{28}}+(8 l+4 w-12) \frac{1}{\sqrt{30}} \\
& +(12 l w-14 l-12 w+14) \frac{1}{\sqrt{32}} \\
= & \frac{3}{2} \sqrt{2} l w+\left(\frac{2}{5} \sqrt{5}+\frac{2}{11} \sqrt{22}+\frac{4}{15} \sqrt{30}+\frac{2}{13} \sqrt{26}-\frac{7}{4} \sqrt{2}\right) l \\
& +\left(-\frac{3}{2} \sqrt{2}+\frac{2}{15} \sqrt{30}+\frac{2}{11} \sqrt{22}+\frac{2}{7} \sqrt{7}\right) w \\
& +\frac{4}{5} \sqrt{5}+\frac{7}{4} \sqrt{2}-\frac{4}{11} \sqrt{22}-\frac{3}{7} \sqrt{7}-\frac{2}{5} \sqrt{30}+\frac{1}{3} \sqrt{6} .
\end{aligned}
$$

\section{Numerical Results and Discussion of Hexagon Star Network}

In this section, we calculate the numerical values for the hexagon star network $\left(G_{l, w}\right)$ by using the ev-degree and ve-degree topological descriptors. We construct the numerical tables of ev-degree and ve-degree descriptors. For different values of $l$ and $w$, we determined the numerical tables for the ev-degree and ve-degree indices.

In this section, we present numerical results related to the ev-degree and ve-degree topological descriptors for the hexagon star network $\left(G_{l, w}\right)$. We have used different values of $l$ and $w$ to compute numerical tables for the ev-degree and ve-degree indices such as the ev-degree Zagreb index, first ve-degree Zagreb $\alpha$ index, first ve-degree Zagreb $\beta$ index, the second ve-degree Zagreb index, ve-degree Randic index, ev-degree Randic index, ve-degree atom-bond connectivity $\left(A \mathbb{B C} \mathbb{V}_{\mathrm{ve}}\right)$ index, ve-degree geometric-arithmetic $\left(\mathbb{G A} \mathbb{A}_{\mathrm{ve}}\right)$ index, ve-degree harmonic $\left(\mathbb{H}_{\mathrm{ve}}\right)$ index, and ve-degree sum-connectivity $\left(\chi_{\mathrm{ve}}\right)$ for the hexagon star network (see Tables 4 and 5). The graphical representation is shown in the Figures 2 and 3. 
TABLE 4: Numerical representation of results.

\begin{tabular}{|c|c|c|c|c|c|}
\hline$(l, w)$ & $\mathbb{M}_{\mathrm{ev}}$ & $\mathbb{M}_{a v e}^{1}$ & $\mathbb{M}_{\beta \mathrm{ve}}^{1}$ & $\mathbb{M}_{\mathrm{ve}}^{2}$ & $\mathbb{R}_{\mathrm{ve}}$ \\
\hline$(5,5)$ & 19440 & 36832 & 9600 & 70808 & 23.465 \\
\hline$(6,6)$ & 27936 & 53408 & 13824 & 103368 & 32.651 \\
\hline$(7,7)$ & 37968 & 73056 & 18816 & 142072 & 43.336 \\
\hline$(8,8)$ & 49536 & 95776 & 24576 & 186920 & 55.521 \\
\hline$(9,9)$ & 62640 & 121568 & 31104 & 237912 & 69.205 \\
\hline$(10,10)$ & 77280 & 150432 & 38400 & 295048 & 84.391 \\
\hline$(11,11)$ & 93456 & 182368 & 46464 & 358328 & 101.08 \\
\hline$(12,12)$ & 111168 & 217376 & 55296 & 427752 & 119.27 \\
\hline$(13,13)$ & 130416 & 255456 & 64896 & 503320 & 138.94 \\
\hline$(14,14)$ & 151200 & 296608 & 75264 & 585032 & 160.13 \\
\hline
\end{tabular}

TABLE 5: Numerical representation of results.

\begin{tabular}{|c|c|c|c|c|c|}
\hline$(l, w)$ & $\mathbb{R}_{\mathrm{ev}}$ & $A B C_{\mathrm{ve}}$ & $\mathbb{G A}_{\mathrm{ve}}$ & $\mathbb{H}_{\mathrm{ve}}$ & $\chi_{\mathrm{ve}}$ \\
\hline$(5,5)$ & 119.95 & 119.83 & 328.05 & 23.281 & 61.757 \\
\hline$(6,6)$ & 169.40 & 168.44 & 465.64 & 32.427 & 86.828 \\
\hline$(7,7)$ & 227.33 & 225.27 & 627.22 & 43.072 & 116.14 \\
\hline$(8,8)$ & 293.75 & 290.30 & 812.79 & 55.218 & 149.69 \\
\hline$(9,9)$ & 368.65 & 363.57 & 1022.4 & 68.864 & 187.49 \\
\hline$(10,10)$ & 452.04 & 445.04 & 1255.94 & 84.009 & 229.53 \\
\hline$(11,11)$ & 543.91 & 534.73 & 1513.6 & 100.65 & 275.82 \\
\hline$(12,12)$ & 644.27 & 632.63 & 1795.2 & 118.80 & 326.34 \\
\hline$(13,13)$ & 753.11 & 738.75 & 2100.7 & 138.45 & 381.11 \\
\hline$(14,14)$ & 870.44 & 853.06 & 2430.2 & 159.59 & 440.11 \\
\hline
\end{tabular}

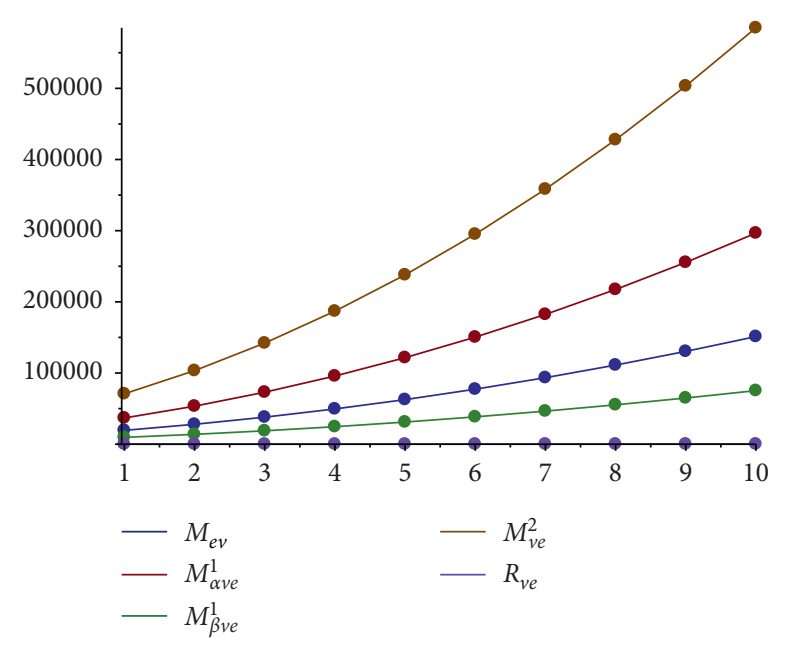

Figure 2: The graphical representation of $\mathbb{M}_{\mathrm{ev}}, \mathbb{M}_{\alpha \mathrm{ve}}^{1}, \mathbb{M}_{\beta \mathrm{ve}}^{1}, \mathbb{M}_{\mathrm{ve}}^{2}$, and $\mathbb{R}_{\mathrm{ve}}$. 


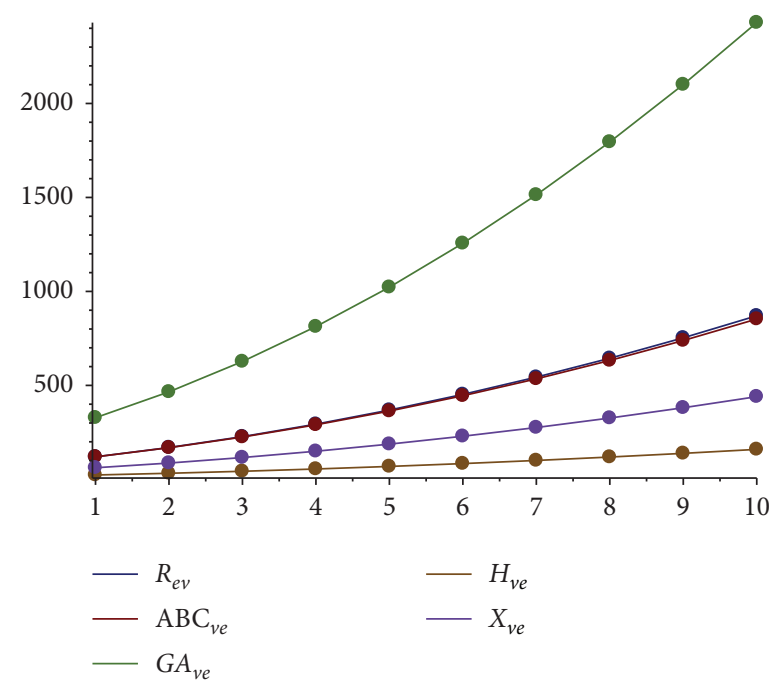

FIGURE 3: The graphical representation of $\mathbb{R}_{\mathrm{ev}}, \mathbb{A} \mathbb{B} \mathbb{C}_{\mathrm{ve}}, \mathbb{G A}_{\mathrm{ve}}, \mathbb{H}_{\mathrm{ve}}$, and $\chi_{\mathrm{ve}}$.

\section{Data Availability}

No data were used to support this study.

\section{Conflicts of Interest}

The authors declare that they have no conflicts of interest.

\section{References}

[1] I. Stojmenovic, Direct Interconnection Networks Parallel and Distributed Computing Handbook, A. Y. Zomaya, Ed., pp. 537-567, McGraw-Hill, Inc., Tokyo, Japan, 1996.

[2] S. Hayat and M. Imran, "Computation of topological indices of certain networks," Applied Mathematics and Computation, vol. 240, pp. 213-228, 2014.

[3] B. F. Begam and J. S. Kumar, "A study on cheminformatics and its applications on modern drug discovery," Procedia Engineering, vol. 38, pp. 1264-1275, 2012.

[4] Z. Raza and M. E. Sukaiti, "M-Polynomial and degree based topological indices of some nanostructures," Symmetry, vol. 12, no. 5, 831 pages, 2020.

[5] M. Bača, J. Horváthová, M. Mokrišová, and A. Suhányiová, "On topological indices of fullerenes," Applied Mathematics and Computation, vol. 251, pp. 154-161, 2015.

[6] H. Wiener, "Structural determination of paraffin boiling points," Journal of the American Chemical Society, vol. 69, pp. 17-20, 1947.

[7] J. Galvez, M. Galvez-Llompart, R. Zanni, and R. GarciaDomenech, "Molecular topology dissimilar similarities," Drug Discovery Today: Technologies, vol. 10, no. 4, pp. e475-e481, 2013.

[8] O. Sporns, "Graph theory methods: applications in brain networks, Dialogues Clin," Neuroscience, vol. 20, pp. 111-121, 2018.

[9] K. H. Rosen, Discrete Mathematics and Its Applications, McGraw-Hill Education, New York, NY, USA, 2018.

[10] S. C. Basak, D. Mills, and M. M. Mumtaz, "Quantitative structure-activity relationship (QSAR) study of dermal absorption using theoretical molecular descriptors, SAR QSAR," Environ. Research, vol. 18, no. 1-2, pp. 45-55, 2007.
[11] M. Arockiaraj, S. Klavžar, J. Clement, S. Mushtaq, and K. Balasubramanian, "Edge distance-based topological indices of strength-weighted graphs and their application to coronoid systems, carbon nanocones and $\mathrm{SiO}_{2}$ nanostructures," Molecular Infrastructure, vol. 38, Article ID 1900039, 2019.

[12] M. Črepnjak and N. Tratnik, "The Szeged index and the Wiener index of partial cubes with applications to chemical graphs," Applied Mathematics and Computation, vol. 309, pp. 324-333, 2017.

[13] A. Ahmad, "Computing the topological descriptors of line graph of the complete m-ary trees," Journal of Intelligent and Fuzzy Systems, vol. 39, no. 1, pp. 1081-1088, 2020.

[14] E. Estrada, "The ABC matrix," Journal of Mathematical Chemistry, vol. 55, no. 4, pp. 1021-1033, 2017.

[15] N. Zahra, M. Ibrahim, and M. K. Siddiqui, "On topological indices for swapped networks modeled by optical transpose interconnection system," IEEE Access, 2020.

[16] T. Vetrk, "Degree-based topological indices of hexagonal nanotubes," Journal of Applied Mathematics and Computing, vol. 58, no. 1, pp. 111-124, 2018.

[17] A. Ahmad, "On the degree based topological indices of benzene ring embedded in P-type-surface in 2D network," Hacettepe Journal of Mathematics and Statistics, vol. 47, no. 1, pp. 9-18, 2018.

[18] S. Zaman, F. A. Abolaban, A. Ahmad, and M. A. Asim, "Maximum $\mathrm{H}$-index ofbipartite network with some given parameters," AIMS Mathematics, vol. 6, no. 5, pp. 5165-5175, 2021.

[19] G. Hong, Z. Gu, M. Javaid, H. M. Awais, and M. K. Siddiqui, "Degree-based topological invariants of metal-organic networks," IEEE Access, vol. 8, pp. 68288-68300, 2020.

[20] M. Ajmal, W. Nazeer, M. Munir, S. M. Kang, and Y. C. Kwun, "Some algebraic polynomials and topological indices of generalized prism and toroidal polyhex networks," Symmetry, vol. 9, no. 1, 5 pages, 2017.

[21] M. K. Siddiqui, M. Imran, and A. Ahmad, "On Zagreb indices, Zagreb polynomials of some nanostar dendrimers," Applied Mathematics and Computation, vol. 280, pp. 132-139, 2016.

[22] M. R. Farahani, W. Gao, M. R. R. Kanna, R. P. Kumar, and J.-B. Liu, "General Randić, sum-connectivity, hyper-Zagreb and harmonic indices, and harmonic polynomial of molecular graphs, Advances in Physical Chemistry," vol. 2016, Article ID 2315949, 6 pages, 2016. 
[23] J. B. Liu, Z. Raza, and M. Javaid, "Zagreb connection numbers for cellular neural networks, Discrete Dynamics in Nature and Society," vol. 2020, Article ID 8038304, 8 pages, 2020.

[24] M. Chellali, T. W. Haynes, S. T. Hedetniemi, and T. M. Lewis, "On ve-degree and ev-degrees in graphs," Discrete Mathematics, vol. 340, no. 2, pp. 31-38, 2017.

[25] B. Horoldagva, K. C. Das, and T. A. Selenge, "On ve-degree and ev-degree of graphs," Discrete Optimatics, vol. 31, pp. 1-7, 2019.

[26] S. Ediz, "A new tool for QSPR researches: degree Randic index," Celal Bayar University Journal of Science, vol. 13, no. 3, pp. 615-618, 2017.

[27] S. Ediz, "On ve-degree molecular topological properties of silicate and oxygen networks," International Journal of Applied Mathematics and Computer Science, vol. 9, no. 1, pp. 1-12, 2018.

[28] B. Sahin and S. Ediz, "On ev-degree and ve-degree topological indices," Iranian Journal of Mathematical Chemistry, vol. 9, no. 4, pp. 263-277, 2018.

[29] J. Zhang, M. K. Siddiqui, A. Rauf, and M. Ishtiaq, "On evdegree and ve-degree based topological properties of single walled titanium dioxide nanotube," Journal of Cluster Science, 2020.

[30] A. Ahmad, "Comparative study of ev-degree and ve-degree topological descriptors for benzene ring embedded in P-typesurface in 2D network," Polycyclic Aromatic Compounds, .

[31] N. A. Koam, A. Ahmad, and M. F. Nadeem, "Comparative study of valency-based topological descriptor for hexagon star network," Computer Systems Science and Engineering, vol. 36, no. 2, pp. pp.293-306, 2021.

[32] N. A. Koam and A. Ahmad, "Polynomials of degree-based indices for three dimensional mesh network, Computers," Materials and Continua, vol. 65, no. 2, pp. 1271-1282, 2020. 